\title{
Porous Sulfur/Carbon Composite Role in Solving the Limitations of Li-S batteries: A Study Case
}

\author{
A. A. M. El-Amir*, A. A. El-Maddah*, M. Hakim, Y.M.Z. Ahmed, E. M.M. Ewais \\ Refractory and Ceramic Materials Dept., Central Metallurgical Research and Development Institute, Cairo, Egypt \\ *Corresponding author: E-mail: elamirahmed.ahmed@gmail.com
}

Received 27 July 2021

Revised 26 August 2021

Accepted for publication 6 October 2021

Published online 12 October 2021

\begin{abstract}
In recent years, $\mathrm{Li}$-ion batteries have become the most widespread among rechargeable battery systems as they possess the highest energy density and specific energy. Among green energy storage devices, lithium-sulfur battery is an important type of Li-ion batteries and is expected to play a leading role in the development of such devices. However, the efficiency of lithium-sulfur batteries has been restricted by the dissolution of polysulfides in the electrolyte and also the poor electrical conductivity of sulfur. The use of porous sulfur/carbon composite in Li-S batteries, has restricted these drawbacks and improved the overall electrochemical and cycling performance of these batteries via enhancing the electrical conductivity of sulfur electrode, trapping the dissolved sulfides and suppressing its migration in the electrolyte, increasing electrolyte and $\mathrm{Li}^{+}$ions transport as well as raising sulfur loading. Hence, this study case focused on the drawbacks of Li-S batteries and their respective solutions suggested in the previous works via the utilization of porous carbon/sulfur cathode.
\end{abstract}

Keywords: Li-S batteries, porous sulfur/carbon nanocomposites, shuttle phenomena, discharge capacity.

\section{Introduction}

Electrical energy storage technologies are currently receiving great interest due to their ability to minimize the consumption of fossil fuels in numerous applications that can reduce environmental pollution to the farthest extent [1-3]. Li-ion batteries with their high energy density that exceeds other rechargeable battery systems are widely spread and have been utilized in numerous applications such as hybrid electric vehicles (HEVs), electric vehicles (EVs), and portable electronic devices [4], [5]. The practical energy density of a Liion battery that has graphite as an anode and $\mathrm{LiCoO}_{2}$ as a cathode, can reach $150 \mathrm{~W} \mathrm{~h} \mathrm{~kg}^{-1}$ in single cells [6]. Despite the constantly growing performance of Li-ion batteries in energy and power density, which is related to the evolution of portable electronics, it has been indicated that these batteries might have reached their limit. Particularly, the energy requirements of the evolved generation of HEVs and EVs might not be satisfied by the Li-ion technology [4], [7]. Therefore, sulfur has been introduced as a cathode to increase the limit of the energy density and achieve other factors such as cost reduction and environmental benefits [1].
Lithium-sulfur batteries (LSBs) which use elemental sulfur as a cathode, have proved their efficiency as energy storage systems with their high specific energy $2500 \mathrm{Wh} / \mathrm{g}$ and specific capacity of $1675 \mathrm{mAh} / \mathrm{g}$. This insertion of elemental sulfur in LSBs has further raised the economic and environmental value of LSBs due to their abundant nature, low cost, and non-toxicity [8]. Nevertheless, Li-S batteries have revealed obstacles related to the sulfur as a cathode material; such as lower operating voltage during discharge $(2.15 \mathrm{~V})$, the isolating characteristics of sulfur and $\mathrm{Li}_{2} \mathrm{~S}$, and the volume change of approximately $79 \%$ that depends on the full conversion of sulfur to $\mathrm{Li}_{2} \mathrm{~S}$ [8-13].

To overcome the drawbacks of LSBs, the electronic conductivity of the sulfur cathode has been further improved by finding a sulfur composite that can act as a cathode and simultaneously restrict the volume variation of sulfur. In this respect, carbon materials are a promising candidate for further improvement in the electrochemical performance of the LSBs due to their superior electrical conductivity. 
Such a feature has allowed carbon materials to compete as an ideal additive for the nonconductive sulfur [4]. In this regard, the present overview explains the limitations of Li-S batteries and clarifies a concise study of the previous works that introduced potential solutions for these drawbacks by involving porous carbon/sulfur composite material as a cathode electrode.

\section{Lithium-Sulfur Batteries}

Conventional components of $\mathrm{Li}-\mathrm{S}$ cell are anodelike metallic lithium, electrolyte with an organic nature, and a sulfur composite as a cathode. The operating principle of these batteries involves two reactions. A discharge reaction that based on the oxidization of the lithium metal at the negative electrode (anode) to create electrons and lithium ions. The positive electrode (cathode) receives the generated lithium ions through the electrolyte inside and receives the electrons through the outer electrical circuit, and therefore the generation of electrical current is achieved. The other reaction involves the reduction of sulfur that occurs when the lithium ions and electrons transfer to the positive electrode which generates the lithium sulfide [14]. Fig. 1 clarifies the charge and discharge processes in the $\mathrm{Li}$ S battery.

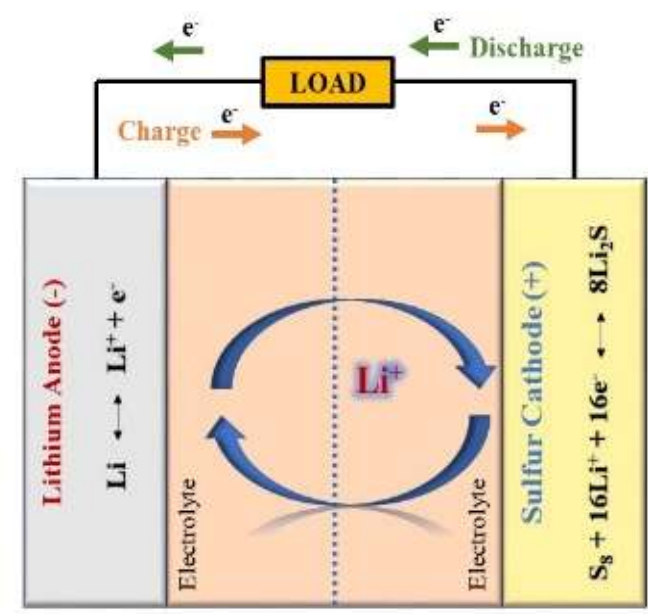

Charge: $8 \mathrm{Li}_{2} \mathrm{~S} \rightarrow \mathrm{S}_{9}+16 \mathrm{Li}$

Discharge: $\mathrm{S}_{8}+16 \mathrm{Li} \longrightarrow 8 \mathrm{Li}_{2} \mathrm{~S}$

Fig. 1 Simplified schematic of the lithium-sulphur battery and the related reactions.

\section{Limitations of lithium-sulfur batteries}

The main problems of these batteries are assigned to the low electric conductivity of sulfur and the reactions of the sulfur electrode that cause volume expansion which in turn destroy the electrode structure. In addition, an active mass loss and shuttle effect are occurred due to the easy dissolution of polysulfide in the electrolyte[15], [16], [14]. Polysulfide dissolution into the electrolyte is the main cause of the shuttle effect. The solid $\mathrm{Li}_{2} \mathrm{~S} / \mathrm{Li}_{2} \mathrm{~S}_{2}$ particles are oxidized during the charging process into long-chain polysulfides. The diffusion force at the end of the charging process is stronger than the electric field force, hence, the long-chain polysulfides are diffused to the anode side. Since the metal anode $\mathrm{Li}$, has a reduction property; the long-chain polysulfides are reacted through a disproportional reaction with Li. Under the electric field force, short-chain polysulfide intermediates are returned to the cathode side. Whereas, the remaining polysulfides are reduced directly to insoluble $\mathrm{Li}_{2} \mathrm{~S} / \mathrm{Li}_{2} \mathrm{~S}_{2}$ particles that cover the Li metal surface. This in turn leads to corrosion of the metal anode $\mathrm{Li}$, loss of active material, and low Coulombic efficiency [17], [8]. Therefore, modifying the cathode material has been spotlighted by researchers, especially respecting the sulfur/carbon composite. The limitations of Li-S batteries can be overcome by involving porous sulfur/carbon composite as a cathode electrode which in turn contributes to enhancing the capability of these batteries by improving their electrochemical properties and preventing the polysulfide shuttle phenomenon.

\section{Porous Sulfur/Carbon Composite as a}

\section{Solution}

The performance of Li-S batteries is highly dependent on the mass and electron transfer in electrochemical reactions, which are affected by various factors such as sulfur loading, surface modifications, and the pore structure [18]. Carbon materials with their excellent conductivity have contributed significantly to the improvement of the electronic conductivity of the sulfur electrode. In addition, the high porosity of carbon-based structure has allowed the effective loading of sulfur, which can hold back polysulfide diffusion, stabilize the volume differences of the electrode, and decrease the shuttle effect [19], [20].

\subsection{Carbon-based materials and their} contribution to improving the performance of Li-

\section{$S$ batteries}

Based on the pore size (L), porous carbon materials are divided into microporous $(\mathrm{L}<2 \mathrm{~nm})$, mesoporous ( $2 \mathrm{~nm}>\mathrm{L}<50 \mathrm{~nm})$, and macroporous ( $\mathrm{L}>50 \mathrm{~nm})$ materials. Each type of these carbon materials has its unique morphological figure-of-merits. The micropore 
carbons are ideal reservoirs to accommodate and immobilize the sulfur material. The mesoporous carbon provides a good opportunity for $\mathrm{Li}+$ ions and electrolytes to transport quickly and easily, in addition to increasing sulfur loading capacity. The macropore carbons act as absorbing channels for liquid electrolytes; and this, in turn, results in the trapping of the dissolved polysulfides leading to significant suppression of their migration. These three types of carbon materials offer at least one of the above benefits to enhance cycling performance when added to the sulfur electrode.

For instance, mesoporous carbon (MC) was prepared by Li et al. [18] with different pore sizes containing sulfur as a composite electrode for $\mathrm{Li}-\mathrm{S}$ batteries. According to this study, partial filling of pores with sulfur (as appears in. Fig 2) has modified the electrochemical properties of the pristine material giving rise to a composite with a capacity of $1390 \mathrm{~mA}$ h $\mathrm{g}^{-1}$ and $840 \mathrm{~mA} \mathrm{~h} \mathrm{~g}{ }^{-1}$ after 100 cycles. Such improvement is related to the electrical contact between the mesoporous carbon and sulfur that subsequently reduced the polysulfide dissolution/diffusion phenomena giving rise to a stable supply of lithium ions.

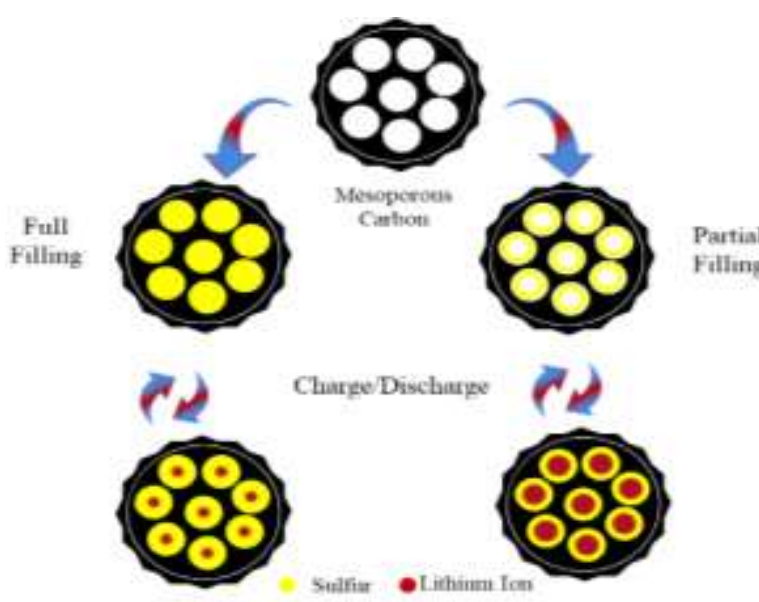

Fig. 2 Schematic illustration of the partial and full filling of mesoporous carbon with sulfur for cathode electrode in lithium sulfur batteries. (Reproduced from reference [18]).

The outcomes of this research indicated that the sulfur loading significantly affected the specific capacity values. $42 \mathrm{wt}$.\% loading of sulfur has shown a capacity with $900 \mathrm{mAh} \mathrm{g}^{-1}$, whereas $51 \mathrm{wt} \%$ loadings shown a capacity with $300 \mathrm{mAh} \mathrm{g}^{-1}$. Such an effect can be clarified by the higher volume expansion and lower conductivity that comes with a higher sulfur load. Thermal treatment of polyacrylonitrile with salt was achieved by Lai et al. [21] to obtain a highly porous carbon (HPC) material, which subsequently was used in the syntheses of sulfur-HPC composite. The specific capacity of the composite with $57 \%$ sulfur delivered was indicated at the beginning to be $1115 \mathrm{mAh} \mathrm{g}^{-1}$ and after 84 cycles was $745 \mathrm{mAh} \mathrm{g}^{-1}$. The authors have confirmed that the shuttle phenomena in Li-S batteries can be effectually prevented upon using HPC material. Fig 3. represents the electrochemical reaction proposed for the sulfur/HPC composite, where it can be inferred that the border between the porous carbon and sulfur was the place where the initial irreversible reaction may occur. This deduced explanation is related to the transfer of electrons that is more favored to occur from conductive porous carbon to sulfur. The next cycle is related to the charge/discharge process which coincides with the transition between low order polysulfides and lithium sulfide and high order polysulfides.

In another work, carbon spheres with narrow micropores have been used by Zhang et al. [22] in an attempt to improve the electrochemical stability of sulfur cathode. In such work, the sulfur was encapsulated in these carbon spheres via the typical procedures shown in the schematic in Fig. 4.

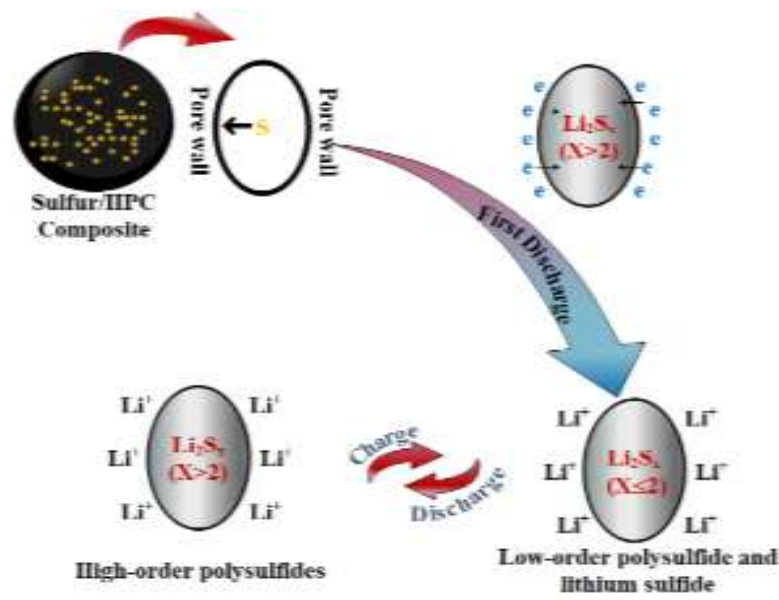

Fig. 3 The electrochemical reaction proposed for the sulfur/HPC composite. (Reproduced from [21]).

The formation of sulfur and polysulfide during the discharge-charge reaction was successfully restricted by the highly porous sulfur-carbon composite prepared by Guo et al. [23]. This restriction was attributed to a large number of pores of approximately $\sim 4 \mathrm{~nm}$ in the prepared cathode. The prepared S-C cathode in such work demonstrated an outstanding electrochemical performance of about $589 \mathrm{~mA} \mathrm{~h} \mathrm{~g}^{-1}$ reversible discharge capacity after 100 cycles under $400 \mathrm{mAg}^{-1}$ current density. Further, a composite of porous carbon nanofiber-sulfur (CNF-S) was prepared by Ji et al. [24] 


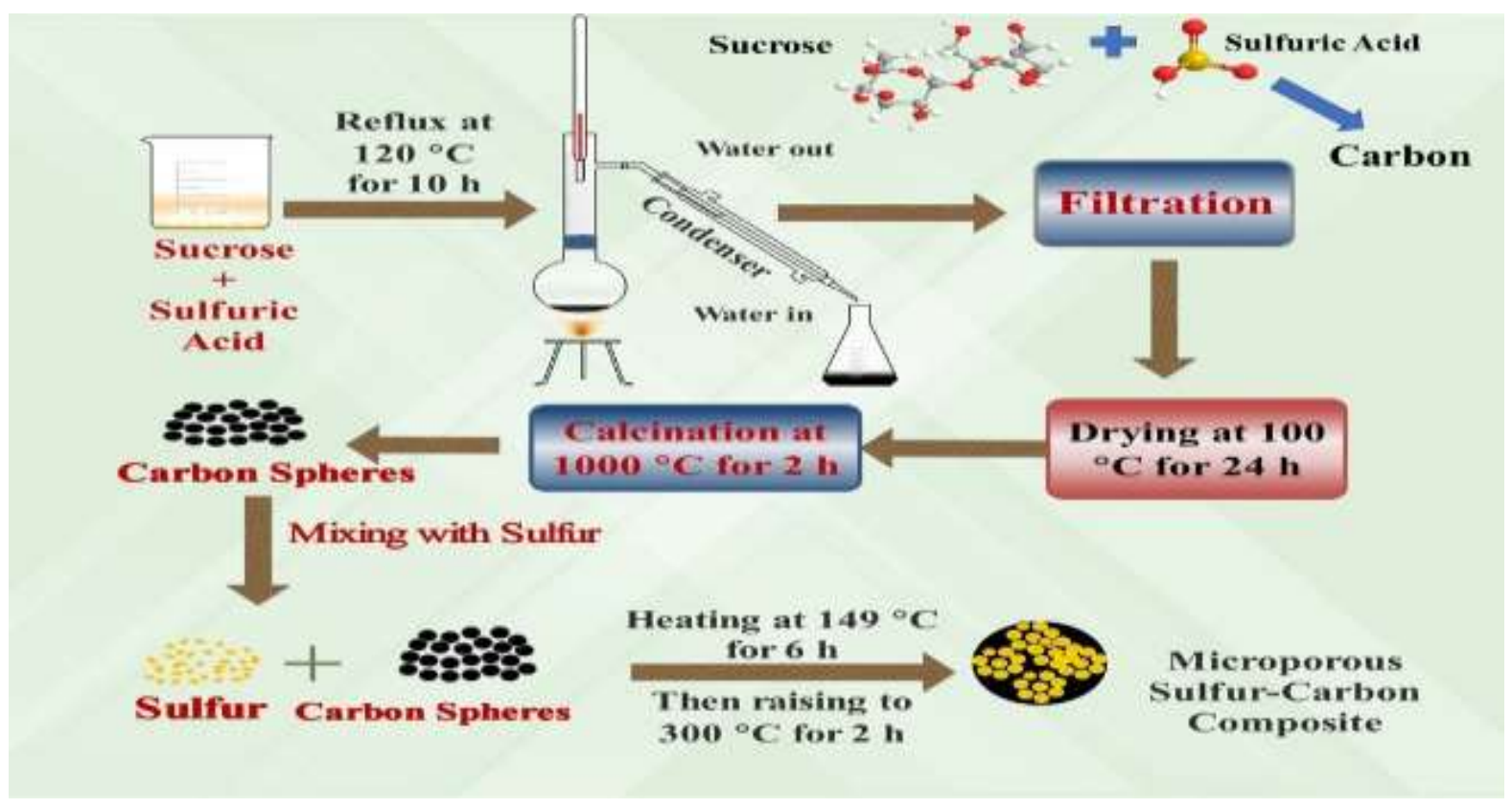

Fig. 4 Typical procedures of encapsulation of sulfur into microporous carbon for preparing sulfur-carbon composite for lithium-sulfur batteries.

through the loading of elemental sulfur into porous carbon nanofiber (CNFs) via chemical solution reaction-deposition technique. The porous CNFs were prepared by electrospinning technique and acted as absorbents to hold back the polysulfides and minimize their dissolution into the electrolyte. Fig. 5 illustrated the typical procedure of CNF-S nanocomposite preparat ion and $\mathrm{Li} / \mathrm{S}$ cell assembling. The as-prepared CNF-S composite with 42 wt.\% sulfur loading demonstrated a discharge capacity of

$1400 \mathrm{mAh} \mathrm{g}^{-1}$ with high retention capacity of approximately $\sim 85 \%$ after 20 cycles at a rate $0.1 \mathrm{C}$, demonstrating its great importance to be utilized as a high-performance cathode for the $\mathrm{Li} / \mathrm{S}$ cells. Kim et al. [25] have elucidated the importance of porous structure in enhancing the performance of Li-S batteries. The modified S-C composite of an open pore structure and $68 \%$ of sulfur loading demonstrated $1000 \mathrm{mAh} \mathrm{g}^{-1}$ initial capacity, $417 \mathrm{Wh} \mathrm{kg}^{-1}$ specific energy, and 623 $\mathrm{Wh} / \mathrm{L}$ energy density at a rate of $0.1 \mathrm{C}$, with $80 \%$ retention capacity after 200 cycles.

By screening several studies [26-33], it can be stated that porous sulfur/carbon composite has maintained an obvious role in improving the performance of $\mathrm{Li}-\mathrm{S}$ batteries and even maintaining great capacity after hundreds of cycles. Hence, a future prospect will contain further investigation on adjusting the porous structure of sulfur/carbon composite, followed by applying various technologies to achieve high porosity sulfur/carbon electrode with highly stable capacity retention.
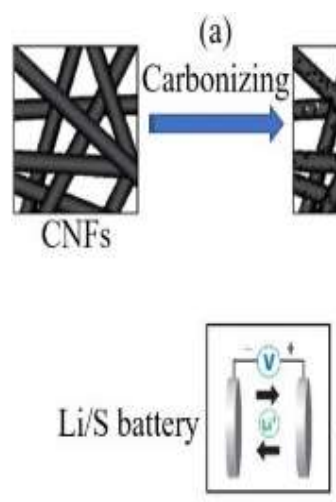

(c) Thermal treatment

(b)

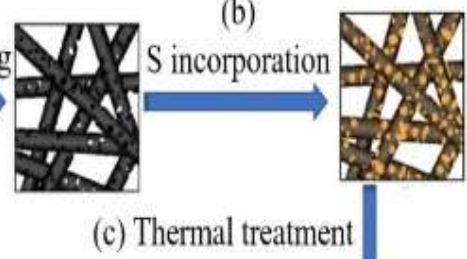

(d)

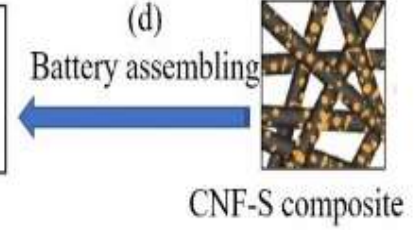

Fig. 5 Schematic of typical experimental procedure. (a) Carbonization of CNFs to get porous CNFs; (b) Incorporation of $\mathrm{S}$ into porous CNFs to produce CNF$\mathrm{S}$ nanocomposite; (c) Thermal treatment to remove sulfur outside of the pores; (d) $\mathrm{Li} / \mathrm{S}$ cell assembling to assess the electrochemical performance of the synthesized CNF-S electrode (Reproduced from [24]).

\section{Conclusion and future prospects}

Recently, various strategies have been made to enhance the electrochemical performance of the $\mathrm{Li} / \mathrm{S}$ batteries and solve their limitations. One of the most important strategies is to develop a porous carbon/sulfur electrode. This study case has summarized the main disadvantages of lithium/sulfur batteries that have restricted their development and offered the role of porous sulfur/carbon composite in overcoming these limitations. It was concluded that the low electrical conductivity of sulfur and easy 
dissolution of polysulfide in the electrolyte can be identified as the main impediments in these batteries. These barriers were overcome by using porous sulfur/carbon composite as a cathodic electrode. Earlier works have brought out the following points:

1- During the discharge-charge reaction, the formation of sulfur and polysulfide can be restricted by the sulfur-carbon composite

2- The partial filling of pores with sulfur can improve the performance of sulfur/carbon composite by improving its electrochemical properties.

3- The amount of loaded sulfur has a significant effect on the discharge capacity of the sulfur/carbon composite

4- Porous carbon materials have contributed to enhancing the electrochemical and cycling performance of $\mathrm{Li} / \mathrm{S}$ batteries via the following functions: (1) acting as potential absorbing channels for electrolyte; (2) trapping the dissolved polysulfides and preventing its migration; (3) immobilizing and accommodating the active material; (4) accelerating the $\mathrm{Li}^{+}$ions and electrolyte transport; (5) improving the conductivity of sulfur electrodes.

Despite the big efforts exerted so far during the recent years for overcoming the limitations of the current Li/S batteries, many issues are still contradictory and require further in-depth research. There are still a lot of challenges in the design and the entire structure of $\mathrm{Li} / \mathrm{S}$ batteries needs to be addressed to become competitive with state-of-the-art $\mathrm{Li}$-ions batteries and tackle the future energy demand. So far, all research on $\mathrm{Li} / \mathrm{S}$ batteries is still at a laboratory level. That's why, more research based on battery components, performance, cycle life, structure, and configuration is demanded to realizing a commercial Li/S battery.

\section{References}

[1] S. Evers, L. F. Nazar, New Approaches for High Energy Density Lithium-Sulfur Battery Cathodes, Acc. Chem. Res. 46 (2013) 1135-1143. doi: $10.1039 / \mathrm{c} 3 \mathrm{sc} 52789 \mathrm{a}$

[2] B. Liu, R. Fang, D. Xie, W. Zhang, H. Huang, Y. Xia, X. Wang, X. Xia, J. Tu, Revisiting scientific issues for industrial applications of lithium-sulfur batteries, Energy Environ. Mater. 1 (2018) 196208. https://doi.org/10.1002/eem2.12021

[3] Z. W. Seh, H. Wang, N. Liu, G. Zheng, W. Li, H. Yao, Y. Cui, High-capacity $\mathrm{Li}_{2} \mathrm{~S}$-graphene oxide composite cathodes with stable cycling performance, Chem. Sci. 5 (2014) 1396-1400. doi: 10.1039/C3SC52789A

[4] T. Li, X. Bai, U. Gulzar, Y. J. Bai, C. Capiglia, W. Deng, X. Zhou, Z. Liu, Z. Feng, R. Proietti Zaccaria, A Comprehensive Understanding of Lithium-Sulfur Battery Technology, Adv. Funct. Mater. $\quad 29 \quad$ (2019) $1-56$. doi:10.1002/adfm.201901730.

[5] W. Guo, A. Bhargav, J. D. Ackerson, Y. Cui, Y. Ma, Y. Fu, Mixture is better: enhanced electrochemical performance of phenyl selenosulfide in rechargeable lithium batteries, Chem. Commun. 54 (2018) 8873-8876. doi:10.1039/c8cc04076a.

[6] R. Marom, S. F. Amalraj, N. Leifer, D. Jacob, D. Aurbach, A review of advanced and practical lithium battery materials, J. Mater. Chem. 21 (2011) 9938-9954. doi:10.1039/c0jm04225k.

[7] A. Fotouhi, D. J. Auger, K. Propp, S. Longo, M. Wild, A review on electric vehicle battery modelling: From Lithium-ion toward LithiumSulphur, Renew. Sustain. Energy Rev. 56 (2016) 1008-1021. doi:10.1016/j.rser.2015.12.009.

[8] H. Dunya, Nanostructured sulfur composite cathodes for lithium- sulfur batteries, Illinois Institute of Technology, 2018.

[9] A. Fedorková, R. Oriňáková, O. Čech, M. Sedlař́ková, New composite cathode materials for li/s batteries: A review, Int. J. Electrochem. Sci. 8 (2013) 10308-10319.

[10] P. G. Bruce, S. A. Freunberger, L. J. Hardwick, J. M. Tarascon, $\mathrm{Li}-\mathrm{O} 2$ and $\mathrm{Li}-\mathrm{S}$ batteries with high energy storage, Nat. Mater. 11 (2012), 19-29. https://doi.org/10.1038/nmat3191

[11] M. K. Song, E. J. Cairns, Y. Zhang, Lithium/sulfur batteries with high specific energy: old challenges and new opportunities, Nanoscale 5 (2013) 2186-2204. DOI: $\underline{10.1039 / C 2 N R 33044 J}$

[12] X. Ji, L. F. Nazar, Advances in Li-S batteries, J. Mater. Chem. 20 (2010) 9821-9826. DOI: $\underline{10.1039 / B 925751 \mathrm{~A}}$

[13] R. Fang, S. Zhao, Z. Sun, D. W. Wang, H. M. Cheng, F. Li, More Reliable Lithium-Sulfur Batteries: Status, Solutions and Prospects, Adv. $\begin{array}{llll}\text { Mater. } & 29 & \text { (2017) } & \text { 1-25. }\end{array}$ https://doi.org/10.1002/adma.201606823

[14] A. Manthiram, Y. Fu, S. Chung, C. Zu, Y. Su, Rechargeable Lithium - Sulfur Batteries, Chem. Rev. 114 (2014) 11751-11787.

[15] J. Li, Y. Huang, S. Zhang, W. Jia, X. Wang, Y. Guo, D. Jia, L. Wang, Decoration of Silica Nanoparticles on Polypropylene Separator for 
Lithium-Sulfur Batteries, ACS Appl. Mater. Interfaces. 9 (2017) 7499-7504. doi:10.1021/acsami.7b00065.

[16] K. Xie, K. Yuan, K. Zhang, C. Shen, W. Lv, X. Liu, J. G. Wang, B. Wei, Dual Functionalities of Carbon Nanotube Films for Dendrite-Free and High Energy-High Power Lithium-Sulfur Batteries, ACS Appl. Mater. Interfaces. 9 (2017) 4605-4613. doi:10.1021/acsami.6b14039.

[17] X. Yang, X. Li, K. Adair, H. Zhang, X. Sun, Structural Design of Lithium-Sulfur Batteries: From Fundamental Research to Practical Application, Springer Singapore, 2018. doi:10.1007/s41918-018-0010-3.

[18] X. Li, Y. Cao, W. Qi, L. V. Saraf, J. Xiao, Z. Nie, J. Mietek, J. G. Zhang, B. Schwenzer, J. Liu, Optimization of mesoporous carbon structures for lithium-sulfur battery applications, J. Mater. Chem. $21 \quad$ (2011) 16603-16610 doi:10.1039/c1jm12979a.

[19] Z. Chang, B. Ding, H. Dou, J. Wang, G. Xu, X. Zhang, Hierarchically Porous Multilayered Carbon Barriers for High-Performance $\mathrm{Li}-\mathrm{S}$ Batteries, Chem. - A Eur. J. 24 (2018) 3768-3775.

[20] S. Yan, X. Yan, Y. Tong, M. Zhang, J. Liu, Application of magnetron sputtering technique to fabricate sulfur/carbon composites cathode plates of lithium-sulfur battery, Mater. Lett. 273 (2020) 127888. doi:10.1016/j.matlet.2020.127888.

[21] C. Lai, X. P. Gao, B. Zhang, T. Y. Yan, Z. Zhou, Synthesis and electrochemical performance of sulfur/highly porous carbon composites, J. Phys. $\begin{array}{lllll}\text { Chem. } & C & 113 & \text { (2009) 4712-4716. }\end{array}$ doi:10.1021/jp809473e.

[22] B. Zhang, X. Qin, G. R. Li, X. P. Gao, Enhancement of long stability of sulfur cathode by encapsulating sulfur into micropores of carbon spheres, Energy Environ. Sci. 3 (2010) 15311537.

[23] L. Li, L. Y. Li, X. D. Guo, B. H. Zhong, Y. X. Chen, Y. Tang, Synthesis and electrochemical performance of sulfur-carbon composite cathode for lithium-sulfur batteries, J. Solid State Electrochem. $17 \quad$ (2013) 115-119. doi:10.1007/s10008-012-1864-7.

[24] L. Ji, M. Rao, S. Aloni, L. Wang, E. J. Cairns, Y. Zhang, Porous carbon nanofiber-sulfur composite electrodes for lithium/sulfur cells, Energy
Environ. $\quad$ Sci. 4 (2011) 5053-5059. doi:10.1039/c1ee02256c.

[25] C. S. Kim, A. Guerfi, P. Hovington, J. Trottier, C. Gagnon, F. Barray, A. Vijh, M. Armand, K. Zaghib, Importance of open pore structures with mechanical integrity in designing the cathode electrode for lithium-sulfur batteries, J. Power Sources. $241 \quad$ (2013) 554-559. doi:10.1016/j.jpowsour.2013.05.026.

[26] Han, Sang-Cheol, et al., Effect of multiwalled carbon nanotubes on electrochemical properties of lithium/sulfur rechargeable batteriesJ. Electrochem. Soc. 150 (2003) A889.

[27] L. Yuan, H. Yuan, X. Qiu, L. Chen, W. Zhu, Improvement of cycle property of sulfurcoated multi-walled carbon nanotubes composite cathode for lithium/sulfur batteries, J. Power Sources 189 (2009) 1141-1146.

[28] W. Wei, J. Wang, L. Zhou, J. Yang, B. Schumann, Y. $\mathrm{Nu} \mathrm{Li,CNT}$ enhanced sulfur composite cathode material for high rate lithium battery, Electrochem. Commun. 13 (2011) 399-402.

[29] J.J. Chen, Q. Zhang, Y. N. Shi, L.L. Qin, Y. Cao, M.S. Zheng, Q.F. Dong, A hierarchical architecture S/MWCNT nanomicrosphere with large pores for lithium sulfur batteries, Phys. Chem. Chem. Phys. 14 (2012) 5376-5382.

[30] Y.J. Choi, K.W. Kim, H.J. Ahn, J.H. Ahn, Improvement of cycle property of sulfur electrode for lithium/sulfur battery, J. Alloys compd. 449 (2008) 313-316.

[31] A.K. Geim, K.S. Novoselov, The rise of graphene. In Nanoscience and technology: a collection of reviews from nature journals, (2010) 11-19.

[32] J.L. Wang, J. Yang, J.Y. Xie, N.X. Xu, Y. J. E. C. $\mathrm{Li}$, Sulfur-carbon nano-composite as cathode for rechargeable lithium battery based on gel electrolyte, Electrochem. Commun. 4 (2002) 499502.

[33] X. Ji, K.T. Lee, L.F. Nazar, A highly ordered nanostructured carbon-sulphur cathode for lithium-sulphur batteries, Nat. mater. 8 (2009) 500-506. 